Acta Math. Appl. Sin.11:4(1995), 389-404.

\title{
ON THE OPTIMALITY IN GENERAL SENSE FOR ODD-BLOCK SEARCH
}

\author{
Mu-Fa Chen and Dan-Hua Huang
}

(Beijing Normal University and Fujian Normal University)

\begin{abstract}
In his classical article[3](1953), J.Kiefer introduced the Fibonacci search as a direct optimal method. The optimality was proved under the restriction: the total number of tests is given in advance and fixed. To avoid this restriction, some different concepts of optimality were proposed and some corresponding optimal methods were obtained in [1], [2], [5] and [6]. In particular, the even-block search was treated in [1]. This paper deals with the odd-block search. The main result is Theorem (1.15).
\end{abstract}

\section{Backgrounds And Main Results}

In this section, we first review some necessary preliminaries and then state our main result. The study of optimal search is usually restricted on the unimodal functions.

(1.1)Definition ${ }^{[3]}$. A function $f$ on interval $[0,1]$ is called unimodal if there exists precisely one maximum at a point $c_{f} \in[0,1]$ and the function is strictly monotone on $\left[0, c_{f}\right]$ and $\left[c_{f}, 1\right]$.

Let $\mathscr{F}$ denote the set of all unimodal functions on $[0,1]$. It is obvious that the unimodal functions have the following advantage: Whenever we have had two tests, we can compare the two results and cancel a part of the interval. Next, we consider only the following testing methods.

(1.2)Definition ${ }^{[3,8]}$. A policy (or strategy, or sequential search) $\mathscr{P}$ is such a rule: at the first step, the rule determines a test point $x_{1}=x_{1}(\mathscr{P})$ independent of $f \in \mathscr{F}$; at the $n$-th step, the rule determines the $n$-th test point $x_{n}=x_{n}(\mathscr{P}, f)$ according to the first $(n-1)$ tested points $x_{1}, \cdots, x_{n-1}$ and their results $f\left(x_{1}\right), \cdots, f\left(x_{n-1}\right)$.

An example of policies is the Fibonacci search or fraction method $\mathscr{F}_{n}$. Recall the Fibonacci sequence: $F_{0}=F_{1}=1, F_{n}=F_{n-1}+F_{n-2}, n \geqslant 2$. For fixed $n \geqslant 1$, the policy $\mathscr{F}_{n}$ is defined as follows: Set $x_{1}=x_{1}\left(\mathscr{F}_{n}\right)=F_{n} / F_{n+1}$. Assume that

1991 Mathematics Subject Classification. 90B40.

Key words and phrases. Fibonacci search, golden section search, odd-block search, optimality in general sense.

This work is partially supported by Ying-Tung Fok Educational Foundation and Natural Science Foundation of China 
at the $m$-th step $(1 \leqslant m \leqslant n-1)$, we have eliminated a part of the interval and the remaining interval is $\left[a_{m}, b_{m}\right]$ with a tested point $c_{m}$ inside. Then, we choose $x_{m+1}=x_{m+1}\left(\mathscr{F}_{n}, f\right)=a_{m}+b_{m}-c_{m}$, which is just the mirror image of $c_{m}$ with respect to the middle point of the interval $\left[a_{m}, b_{m}\right]$. In what follows, we call the last procedure the symmetry rule.

Warning. It can be happened that for a given $f$, at some steps the two tested results are the same. And so after the elimination, the remaining interval has no tested point inside. In this case, we have to modify the above $\mathscr{F}_{n}$. Simply regard the remaining interval as our initial testing interval and apply the same rule. But in what follows we may and will omit this exceptional case for saving the space.

Let policy $\mathscr{P}$ act on $f \in \mathscr{F}$ in $n$ times. Among the $n$ tested points, there is one point, denoted by $c_{f}(\mathscr{P}, n)$, at which, $f$ achieves its maximum. Recall the real maximum of $f$ is achieved at $c_{f}$.

(1.3)Definition. We call $\delta(\mathscr{P}, n):=\sup _{f \in \mathscr{F}}\left|c_{f}-c_{f}(\mathscr{P}, n)\right|$ the accuracy of $\mathscr{P}$ at the $n$-th step. We say that a policy $\mathscr{Q}$ is optimal with $n$ steps if for any policy $\mathscr{P}, \delta(\mathscr{P}, n) \geqslant \delta(\mathscr{Q}, n)$.

(1.4)Theorem (J. Kiefer ${ }^{[3]}$ ). The fraction method $\mathscr{F}_{n}$ is optimal with $n$ steps.

At the end of his paper [3], Kiefer noted that it is not convenient in practice to use $\mathscr{F}_{n}$ since we have to decide in advance the precise number $n$ of tests. Because of this reason, Kiefer suggested to use $\omega:=\lim _{n \rightarrow \infty} F_{n} / F_{n+1}=(\sqrt{5}-1) / 2$ as the first testing point $x_{1}$ instead of $F_{n} / F_{n+1}$ and then keep the symmetry rule. The later one is called the golden section search (see also [8]), denoted by $\mathscr{W}$. However, L. K. Hua pointed out that $\mathscr{W}$ is optimal in a different sense and he regarded $\mathscr{F}_{n}$ as an approximation of $\mathscr{W}$.

(1.5)Definition. A policy $\mathscr{P}$ is called symmetric if at the first step, choose $x_{1}=$ $x_{1}(\mathscr{P})$ independent of $f \in \mathscr{F}$. Starting from the second step, choose the new test point according to the symmetry rule.

The next result is due to Hua for symmetric policy and extended by J. W. Hong to the general case.

(1.6) Theorem(Hua ${ }^{[6,7]}$ and Hong $\left.{ }^{[4]}\right)$. For any policy $\mathscr{P}$, we have $\delta(\mathscr{P}, n) \geqslant$ $\delta(\mathscr{W}, n)$ for all sufficiently large $n$. In other words, the policy $\mathscr{W}$ is optimal at infinity.

To understand our optimality in a general sense, recall that for each $n$, the optimal policy $\mathscr{F}_{n}$ gives us the optimal accuracy at the last $(n$-th $)$ step: $\delta\left(\mathscr{F}_{n}, n\right)=$ $1 / F_{n+1}$. By comparing the relative difference (but not the absolute difference since at different steps, the testing intervals have different scale) between a policy $\mathscr{P}$ and the policy $\mathscr{F}_{n}$ at the $n$-th step:

$$
\left(\delta(\mathscr{P}, n)-\delta\left(\mathscr{F}_{n}, n\right)\right) / \delta\left(\mathscr{F}_{n}, n\right)=F_{n+1} \delta(\mathscr{P}, n)-1,
$$

we arrive at the following notion

(1.7)Definition ${ }^{[1]}$. We call $\delta(\mathscr{P}):=\sup _{n \geqslant 1} F_{n+1} \delta(\mathscr{P}, n)$ the accuracy of $\mathscr{P}$. A policy $\mathscr{Q}$ is called optimal if for any policy $\mathscr{P}, \delta(\mathscr{P}) \geqslant \delta(\mathscr{Q})$. 
(1.8) Theorem $^{[1]}$. For any symmetric $\mathscr{P}$, we have $\delta(\mathscr{P}) \geqslant \delta(\mathscr{W})$.

Before moving further, let us introduce some notations which will be used throughout this paper. Let $k_{1}, k_{2}, \cdots\left(k_{1} \geqslant 2\right)$ be a sequence of positive integers. Set

$$
c(k)=\left(\begin{array}{ll}
\chi(k) & {\left[\frac{k+1}{2}\right]} \\
\chi(k+1) & {\left[\frac{k+2}{2}\right]}
\end{array}\right)
$$

where $[x]$ is the integer part of $x$ and $\chi(k)=0$ or 1 according to $k$ being odd or even respectively. Next, let $\left(\begin{array}{l}x_{n} \\ y_{n}\end{array}\right)$ be the solution to the equations

$$
\left(\begin{array}{l}
x_{n} \\
y_{n}
\end{array}\right)=c\left(k_{n+1}\right)\left(\begin{array}{l}
x_{n+1} \\
y_{n+1}
\end{array}\right), \quad n \geqslant 0, \quad x_{0}=1 \text {. }
$$

It was proved in [5] that the solution $\left(\begin{array}{l}x_{n} \\ y_{n}\end{array}\right)$ not only exists but also unique whenever there are infinitely many odd numbers in the sequence $\left\{k_{1}, k_{2}, \cdots\right\}$.

We now fix $n \geqslant 1$ and make the boundary condition at the final (rather than the first) step: $\left(\begin{array}{c}X_{n} \\ Y_{n}\end{array}\right)=\left(\begin{array}{l}1 \\ 2\end{array}\right)$. Define $\left(\begin{array}{c}X_{m} \\ Y_{m}\end{array}\right)=c\left(k_{m+1}\right)\left(\begin{array}{c}X_{m+1} \\ Y_{m+1}\end{array}\right), 0 \leqslant m \leqslant n-1$. In particular, if $k_{m} \equiv 2 i-1$, then $X_{0}=: F_{n+1}^{(i)}$ gives us the generalized Fibonacci sequence ${ }^{[8]}: F_{0}^{(i)}=F_{1}^{(i)}=1$ and $F_{n}^{(i)}=i\left(F_{n-1}^{(i)}+F_{n-2}^{(i)}\right)$ for $n \geqslant 2$. For the special case that $k_{m} \equiv 2 i(i \geqslant 1)$, we rewrite $X_{0}$ as $E_{n}^{(i)}: E_{n}^{(i)}=2(i+1)^{n}-1$.

(1.9)Definition. Given an interval $[a, b]$ and $\alpha, \beta>0$. The partition $a=a_{1}<$ $b_{1}=a_{2}<b_{2}=a_{3}<\cdots<b_{N}=b$ satisfying $b_{2 k-1}-a_{2 k-1}=\alpha$ and $b_{2 k}-a_{2 k}=\beta$ for each $k \geqslant 1$, if exists, is called an $[\alpha, \beta]$-partition.

(1.10)Definition. Fix $n \geqslant 1$ and $k_{1} \geqslant 2, k_{2}, \cdots, k_{n}$. Define the policy $\mathscr{G}_{n}$ as follows. At the first step, we take the $[\alpha, \beta]$-partition with ratio $\alpha / \beta=X_{1} /\left(Y_{1}-X_{1}\right)$ and arrange the $k_{1}$ tests at the dividing points. At the $m$-th step, we choose the $[\alpha, \beta]$-partition with ratio $\alpha / \beta=X_{m} /\left(Y_{m}-X_{m}\right), 2 \leqslant m \leqslant n$, and arrange the $k_{m}$ new tests, plus the tested point left from the previous step, at the dividing points.

(1.11)Theorem (Hong $\left.{ }^{[5]}\right)$. The policy $\mathscr{G}_{n}$ is optimal with $n$ steps. Moreover, $\delta\left(\mathscr{G}_{n}, n\right)=1 / X_{0}$.

(1.12)Definition. A policy $\mathscr{P}$ is called basic if at each step the tests are arranged at the dividing points of an $[\alpha, \beta]$-partition. For the special case that $k_{n} \equiv 2 i$ and $\alpha=\beta$ at each step, the basic policy is denoted by $\mathscr{E}^{(i)}$.

The reason we pay special attention to the basic policies is that all known optimal policies are basic and on the other hand, complicated policies are not useful in practice. Having these preparations in mind, we can return to our main discussion. Suppose that at each step, $k_{m}=2 i(i \geqslant 1)$. Then, it is known that there is no optimal policy at infinity ${ }^{[5]}$. Nevertheless, in our new sense, there does exist an optimal one.

(1.13) Theorem $^{[1]}$. Let $i \geqslant 2$. For any basic policy $\mathscr{P}$, we have $\delta(\mathscr{P}) \geqslant \delta\left(\mathscr{E}^{(i)}\right)=$ $2(i+1) /(2 i+1)$. where the accuracy $\delta(\mathscr{P})$ is defined by Definition (1.7) but replacing $F_{n+1}$ with $E_{n}^{(i)}$. 
The policy $\mathscr{E}^{(i)}$ comes with no surprise since its construction is quite natural. However, the case that $k_{n} \equiv 2$ is excluded from the above theorem. In this case, the optimal policy takes $3 / 7$ and $4 / 7$ as the testing points at the first step and uses the same construction as $\mathscr{E}^{(i)}(i \geqslant 2)$ for the subsequent steps ${ }^{[1]}$.

We now consider the odd-block search. That is, $k_{n} \equiv 2 i-1(i \geqslant 2)$. In this case, for fixed $n$, the optimal policy $\mathscr{G}_{n}$ gives us the $[\alpha, \beta]$-partition: $\alpha=$ $\alpha^{(n)}=F_{n}^{(i)} / F_{n+1}^{(i)}, \beta=\beta^{(n)}=1 / i-\alpha$ for the first step. By the same procedure as we mentioned before, $\omega(i):=\lim _{n \rightarrow \infty} F_{n}^{(i)} / F_{n+1}^{(i)}=(\sqrt{i(i+4)}-i) /(2 i)$, we obtain a basic policy $\mathscr{W}^{(i)}$ by replacing $\alpha=F_{n}^{(i)} / F_{n+1}^{(i)}$ with $\omega(i)$ at the first step. More precisely, at the $m$-th $(m \geqslant 1)$ step, we have the $[\alpha, \beta]$-partition with $\alpha=\alpha_{m}=\omega(i)^{m}$, $\beta=\beta_{m}=\omega(i)^{m+1}$. Moreover, it is easy to check that $\delta\left(\mathscr{W}^{(i)}, n\right)=\omega(i)^{n}, n \geqslant 1$. Furthermore, it was proved ${ }^{[4,5]}$ in a slight different sense that the policy $\mathscr{W}^{(i)}$ is indeed the optimal policy at infinity (See Section 4 for details).

Next, is it true the policy $\mathscr{W}^{(i)}(i \geqslant 2)$ being the optimal one in the general sense? The answer is surprisingly to be negative!

(1.14)Definition. Define a basic policy $\mathscr{H}=\mathscr{H}^{(i)}$ as follows. At the first step, we take the $\left[\alpha_{1}, \beta_{1}\right]$-partition:

$\alpha_{1}=\left\{\frac{1}{i}\left[\frac{i+1}{2}\right]+\chi(i) \omega(i)\right\} \omega(i)=\frac{1}{i}\left\{\chi(i)+(\chi(i-1)-\chi(i))\left[\frac{i+1}{2}\right] \omega(i)\right\}, \quad \beta_{1}=\frac{1}{i}-\alpha_{1}$

and at the $n$-th step, we choose the $\left[\alpha_{n}, \beta_{n}\right]$-partition: $\alpha_{n}=\omega(i)^{n}, \beta_{n}=\omega(i)^{n+1}$, $n \geqslant 2$.

The remainder of this paper is to prove the following result.

(1.15) Theorem. For any basic policy $\mathscr{P}$, we have $\delta(\mathscr{P}) \geqslant \delta(\mathscr{H})$. In other words, the policy $\mathscr{H}=\mathscr{H}^{(i)}$ is optimal in the general sense among the basic policies.

The paper is organized as follows. In the next section, we prove some elementary properties about the generalized Fibonacci sequence and a related sequence. In Section 3 , we study how to compute the accuracy $\delta(\mathscr{P}, n)$. At the end of this section, we explain the main steps of the proof of Theorem (1.15). Especially, we explain why we have to study the optimal policy at infinity, which is the topic studied in Section 4. Having these preparations, the proof of Theorem (1.15) is completed in Section 5. It turns out that the present proof of the main theorem is quite complicated and lengthy but we hope that the work would provide some light to solve the problem for the general situation where the numbers $\left\{k_{n}\right\}$ being arbitrary.

\section{Properties of $F$-Sequence And $G$-Sequence}

From now on, we fix $i \geqslant 2$ and $k_{n}=2 i-1$ at least for all $n \geqslant 2$. Thus, we may drop the superscript $i$ from $\mathscr{W}^{(i)}, F_{n}^{(i)}$ and so on without any confusion.

Recall the $F$-sequence is defined by

$$
F_{0}=F_{1}=1, \quad F_{n}=i\left(F_{n-1}+F_{n-2}\right), \quad n \geqslant 2 .
$$

A related sequence, called $G$-sequence, is defined by

$$
G_{-1}=0, \quad G_{0}=1, \quad G_{n}=i\left(G_{n-1}+G_{n-2}\right), \quad n \geqslant 1 .
$$

Let $\omega=\omega(i)=(\sqrt{i(i+4)}-i) /(2 i)$ which is the positive root of

$$
i\left(\omega+\omega^{2}\right)=1 .
$$


(2.4)Lemma. For the F-sequence, we have

$$
\begin{aligned}
F_{n+1} & =\frac{1}{2}\left\{\left(1+3 \sqrt{\frac{i}{i+4}}\right)\left(\frac{i+\sqrt{i(i+4)}}{2}\right)^{n}+\left(1-3 \sqrt{\frac{i}{i+4}}\right)\left(\frac{i-\sqrt{i(i+4)}}{2}\right)^{n}\right\} \\
& =\frac{1}{2}\left\{\left(1+3 \sqrt{\frac{i}{i+4}}\right) \omega^{-n}+\left(1-3 \sqrt{\frac{i}{i+4}}\right)(-i \omega)^{n}\right\}, \quad n \geqslant-1 .
\end{aligned}
$$

$$
F_{n+1} F_{n-1}-F_{n}^{2}=(2 i-1)(-i)^{n-1}, \quad n \geqslant 1 .
$$

$$
F_{n} F_{n-1}-F_{n+1} F_{n-2}=(2 i-1)(-i)^{n-1}, \quad n \geqslant 2 .
$$

$$
\text { As } n \rightarrow \infty, \frac{F_{2 n-1}}{F_{2 n}} \text { strictly increases to } \omega \text { and } \frac{F_{2 n}}{F_{2 n+1}} \text { strictly decreases to } \omega \text {. }
$$

As $n \rightarrow \infty, \frac{F_{2 n-1}}{F_{2 n+1}}$ strictly increases to $\omega^{2}$ and $\frac{F_{2 n}}{F_{2 n+2}}$ strictly decreases to $\omega^{2}$.

Proof. Clearly, (2.5) follows from (2.1) and (2.3). One may prove (2.6) by using induction and (2.1). Then (2.7) follows from (2.6). Next, by (2.7), we have

$$
\frac{F_{2 n+1}}{F_{2 n+2}}-\frac{F_{2 n-1}}{F_{2 n}}=\frac{(2 i-1)(-i)^{2 n}}{F_{2 n} F_{2 n+2}}>0, \quad \frac{F_{2 n}}{F_{2 n+1}}-\frac{F_{2 n-2}}{F_{2 n-1}}=\frac{(2 i-1)(-i)^{2 n-1}}{F_{2 n-1} F_{2 n+1}}<0 .
$$

From this and (2.1), it is easy to see that (2.8) holds. Similarly, (2.9) follows from (2.1), (2.7) and (2.8).

(2.10)Lemma. For the G-sequence, we have

$$
\begin{aligned}
G_{n} & =\frac{1}{\sqrt{i(i+4)}}\left\{\left(\frac{i+\sqrt{i(i+4)}}{2}\right)^{n+1}-\left(\frac{i-\sqrt{i(i+4)}}{2}\right)^{n+1}\right\} \\
& =\frac{1}{\sqrt{i(i+4)}}\left\{\omega^{-(n+1)}-(-i \omega)^{n+1}\right\}, \quad n \geqslant-1 .
\end{aligned}
$$

$$
G_{n} G_{m}-G_{n+1} G_{m-1}=(-i)^{m} G_{n-m}, \quad n+1 \geqslant m \geqslant 0 .
$$

$$
G_{n} G_{m}-G_{n+2} G_{m-2}=-(-i)^{m} G_{n-m+1}, \quad n+1 \geqslant m \geqslant 1 .
$$

As $n \rightarrow \infty, \frac{G_{2 n-1}}{G_{2 n}}$ strictly increases to $\omega$ and $\frac{G_{2 n}}{G_{2 n+1}}$ strictly decreases to $\omega$.

As $n \rightarrow \infty, \frac{G_{2 n-1}}{G_{2 n+1}}$ strictly increases to $\omega^{2}$ and $\frac{G_{2 n}}{G_{2 n+2}}$ strictly decreases to $\omega^{2}$.

Proof. The proof is similar to the previous one except (2.12) and (2.13). But one may use induction on $m \geqslant 0$ to prove that (2.12) holds for all $m \leqslant n+1$. Then, (2.13) follows from (2.12) and (2.2). 
(2.16)Lemma. Let $a, b, c, d$ be positive numbers and $n, m$ be non-negative integers with $n \geqslant m$. Then

$$
\frac{a G_{m}+b G_{m-1}}{a G_{n+1}+b G_{n}}-\frac{c G_{m}+d G_{m-1}}{c G_{n+1}+d G_{n}}<0 \quad(\text { resp. }>0,=0)
$$

if and only if $(-1)^{m}(a d-b c)<0($ resp. $>0,=0)$.

Proof. Simply use (2.13) .

(2.17)Lemma. For the relation between the F-sequence and $G$-sequence, we have

$$
\begin{aligned}
& F_{n}=G_{n-1}+i G_{n-2}, \quad n \geqslant 1, \\
& F_{n} G_{m}-F_{n+2} G_{m-2}=-(-i)^{m} F_{n-m+1}, \quad n+1 \geqslant m \geqslant 1,
\end{aligned}
$$

$$
\frac{F_{2 n-1}}{F_{2 n+1}}<\frac{G_{2 n-1}}{G_{2 n+1}}<\frac{F_{2 n+1}}{F_{2 n+3}}, \quad \frac{F_{2 n}}{F_{2 n+2}}<\frac{G_{2 n-2}}{G_{2 n}}<\frac{F_{2 n-2}}{F_{2 n}}, \quad n \geqslant 1 .
$$

Proof. The first assertion follows from the definitions of the sequences plus induction. Then, (2.19) follows from (2.18) and (2.13). Finally, (2.20) follows from (2.18) and (2.12).

To conclude this section, we list the first few terms of the sequences for the subsequent use.

$$
\begin{aligned}
& F_{5}=i^{2}\left(2 i^{2}+5 i+1\right), \quad F_{6}=i^{3}\left(2 i^{2}+7 i+4\right), \quad F_{7}=i^{3}\left(2 i^{3}+9 i^{2}+9 i+1\right) . \\
& G_{-1}=0, \quad G_{0}=1, \quad G_{1}=i, \quad G_{2}=i(i+1), \\
& G_{3}=i^{2}(i+2), \quad G_{4}=i^{2}\left(i^{2}+3 i+1\right), \quad G_{5}=i^{3}\left(i^{2}+4 i+3\right) .
\end{aligned}
$$

\section{The Accuracy of $\mathscr{H}$ and $\mathscr{W}$. The Idea of the Main Proof.}

Suppose that a policy $\mathscr{P}$ acts on $f \in \mathscr{F}$, after $n$ steps, the remaining interval is $\left[a_{n}, b_{n}\right]$. If there is a tested point inside of $\left[a_{n}, b_{n}\right]$, then let $c_{n}$ denote this point. Next, set

$$
\Delta(\mathscr{P}, f, n)=b_{n}-a_{n}, \quad \delta(\mathscr{P}, f, n)=\left\{\begin{array}{l}
b_{n}-a_{n}, \text { if there is no } c_{n} \\
\max \left\{c_{n}-a_{n}, b_{n}-c_{n}\right\}, \text { if } c_{n} \text { exists. }
\end{array}\right.
$$

For convenience, put $\Delta(\mathscr{P}, f, 0)=\delta(\mathscr{P}, f, 0)=1$. Define

$$
\Delta(\mathscr{P}, n)=\sup _{f} \Delta(\mathscr{P}, f, n), \quad \delta(\mathscr{P}, n)=\sup _{f} \delta(\mathscr{P}, f, n) .
$$

The last one is the accuracy of $\mathscr{P}$ at the $n$-th step, which is precisely the same as we defined before. Finally, the accuracy of $\mathscr{P}$ is given by $\delta(\mathscr{P})=\sup _{n \geqslant 1} F_{n+1} \delta(\mathscr{P}, n)$, since we are now in the case that $k_{n}=2 i-1$.

As we have seen in the first section, $\delta(\mathscr{W}, n)=\omega^{n}, n \geqslant 0$. We now prove 
(3.1)Lemma. $\delta(\mathscr{W})=F_{2} \omega=2 i \omega$.

Proof. The proof will be done once we show that as $n \rightarrow \infty, F_{2 n+1} \omega^{2 n}$ increases and $F_{2 n+2} \omega^{2 n+1}$ decreases to the same limit

$$
\lim _{n \rightarrow \infty} F_{n+1} \omega^{n}=\frac{1}{2}+\frac{3}{2} \sqrt{\frac{i}{i+4}}=\frac{1}{i+4}(2(i+1)+3 i \omega) .
$$

The last conclusion follows from (2.5) immediately. On the other hand, by (2.9), we have

$$
\frac{F_{2 n+3} \omega^{2 n+2}}{F_{2 n+1} \omega^{2 n}}=\omega^{2} / \frac{F_{2 n+1}}{F_{2 n+3}} \downarrow 1, \quad \frac{F_{2 n+2} \omega^{2 n+1}}{F_{2 n} \omega^{2 n-1}}=\omega^{2} / \frac{F_{2 n}}{F_{2 n+2}} \uparrow 1 .
$$

Hence the proof is completed.

As for the policy $\mathscr{H}$ defined in (1.14), we have

(3.3)Lemma. $\delta(\mathscr{H})=F_{4} \omega^{3}=i^{2}(2 i+3) \omega^{3}=: \delta$.

Proof. The last equality follows from (2.21). By (2.3), we can also express $\delta$ as follows:

$$
\begin{aligned}
\delta & =i(2 i+3)(1-i \omega) \omega=i(2 i+3)((i+1) \omega-1) \\
& =\frac{2 i+3}{2}((i+1) \sqrt{i(i+4)}-i(i+3))<2 .
\end{aligned}
$$

By the definition of $\mathscr{H}$, it is easy to check that

$$
\delta(\mathscr{H}, 1)=\left\{\frac{1}{i}\left[\frac{i+1}{2}\right]+\chi(i) \omega\right\} \omega, \quad \Delta(\mathscr{H}, 1)=1 / i
$$

and $\delta(\mathscr{H}, n)=\omega^{n}, \Delta(\mathscr{H}, n)=\omega^{n-1} / i, n \geqslant 2$. Thus, it follows from the proof of Lemma (3.1) that

$$
\begin{aligned}
\delta(\mathscr{H}) & =\sup _{n \geqslant 1} F_{n+1} \delta(\mathscr{H}, n) \\
& =\max \left\{F_{2} \delta(\mathscr{H}, 1), \sup _{n \geqslant 2} F_{n+1} \delta(\mathscr{H}, n)\right\} \\
& =\max \left\{F_{2} \delta(\mathscr{H}, 1), F_{4} \omega^{3}\right\} .
\end{aligned}
$$

Hence, we need only to show that

$$
2 i\left(\frac{1}{i}\left[\frac{i+1}{2}\right]+\chi(i) \omega\right)<i(2 i+3)(1-i \omega) .
$$

If $i=2$, then $\omega=(\sqrt{3}-1) / 2$. In this case, a direct computation shows that (3.5) holds. For $i \geqslant 3$, the left hand side of (3.5) is less or equal to $i+1+2 i \omega$. Thus, it suffices to show that $i\left(2 i^{2}+3 i+2\right) \omega<2 i^{2}+2 i-1$. Noticing that $\omega<F_{4} / F_{5}=(2 i+3) /\left(2 i^{2}+5 i+1\right)$, the above inequality follows immediately for all $i \geqslant 3$. 
(3.6) Corollary. $\delta(\mathscr{W})>\delta(\mathscr{H})$.

This corollary means that $\mathscr{H}$ is better than $\mathscr{W}$ in the general sense. Comparing $\mathscr{H}$ with $\mathscr{W}$ carefully, we see that the difference between these two policies is only at the first step. For $\mathscr{H}$, we choose

$$
\alpha_{1}(\mathscr{H})=\left\{\frac{1}{i}\left[\frac{i+1}{2}\right]+\chi(i) \omega\right\} \omega, \quad \beta_{1}(\mathscr{H})=1 / i-\alpha_{1}(\mathscr{H}) .
$$

For $\mathscr{W}$, we choose $\alpha_{1}(\mathscr{W})=\omega, \beta_{1}(\mathscr{W})=1 / i-\alpha_{1}(\mathscr{W})$. Starting from the second step, the construction rule for the two policies is completely the same. Now, what is the key point to making such a choice for $\mathscr{H}$. The reason is as follows. Since at the first step, we have an odd-block search $k_{1}=2 i-1$, for any basic policy $\mathscr{P}$, we always have $\Delta(\mathscr{P}, 1)=1 / i$. On the other hand, at the second step, including the tested point (left from the first step), there are altogether $2 i$ tests. However, the $\ell$-th position $(1 \leqslant \ell \leqslant 2 i)$ located by the tested point left from the first step does not make any influences to the construction for the second step, since the key of the construction is the ratio $\alpha_{2} / \beta_{2}$. This is due to the fact that our policies are basic. But the location of the tested point does influence $\delta(\mathscr{P}, 1)$. Furthermore, each basic policy $\mathscr{P}$ corresponds uniquely a basic policy with initial testing interval $[0,1 / i]$ and with testing numbers: $k_{1}=2 i, k_{n}=2 i-1, n \geqslant 2$. Let us denote the later policy by $\mathscr{P}_{1}$. Corresponding to $\mathscr{H}$, we have $\mathscr{H}_{1}$. Conversely, due to the rule for the basic policies, a basic policy $\mathscr{P}_{1}$ with initial testing interval $[0,1 / i]$ and with testing numbers $k_{1}=2 i, k_{n}=2 i-1, n \geqslant 2$, determines uniquely (here we regard those policies which have the same accuracy at the first step as the same) a basic policy $\mathscr{P}$ with initial testing interval $[0,1]$ and with testing numbers $k_{n} \equiv 2 i-1$. Moreover, It is obvious that

$$
\delta(\mathscr{P})=\sup _{n \geqslant 1} F_{n+1} \delta(\mathscr{P}, n)=\sup _{n \geqslant 1} F_{n+2} \delta\left(\mathscr{P}_{1}, n\right) .
$$

In particular,

$$
\delta(\mathscr{H})=\sup _{n \geqslant 1} F_{n+2} \delta\left(\mathscr{H}_{1}, n\right)=F_{4} \omega^{3} .
$$

The above discussions tell us, in order to prove Theorem (1.15), we need only to study the basic polices $\mathscr{P}_{1}$ and proving that

$$
\sup _{n \geqslant 1} F_{n+2} \delta\left(\mathscr{P}_{1}, n\right) \geqslant F_{4} \omega^{3}=\sup _{n \geqslant 1} F_{n+2} \delta\left(\mathscr{H}_{1}, n\right) .
$$

To fix our idea, let us repeat the construction of the basic policy $\mathscr{H}_{1}$. At the $n$-th step, we take the $\left[\alpha_{n}, \beta_{n}\right]$-partition with $\alpha_{n}=\omega^{n+1}, \beta_{n}=\omega^{n}$.

(3.10)Definition. We say that two policies $\mathscr{P}$ and $\mathscr{P}^{\prime}$ are equivalent if for all $n \geqslant 1, \delta(\mathscr{P}, n)=\delta\left(\mathscr{P}^{\prime}, n\right)$.

Again, due to the rule of the basic policies, it is easy to check the following fact:

(3.11)Lemma. If a basic policy $\mathscr{P}_{1}$ is not equivalent to $\mathscr{H}_{1}$, then we must have $\alpha_{1}=\alpha_{1}\left(\mathscr{P}_{1}\right) \neq \omega^{2}=\alpha_{1}\left(\mathscr{H}_{1}\right)$.

We now going to study how to compute the accuracy at each step. 
(3.12)Remark. Let $\left[a_{n}, c_{n}, b_{n}\right]$ be the remaining interval left from the $n$-th step, for the purpose of computing the accuracy at the $(n+1)$-th step, we may and will assume that $a_{n}=0, c_{n}=\delta\left(\mathscr{P}_{1}, n\right), b_{n}=\Delta\left(\mathscr{P}_{1}, n\right)$ and $c_{n} \geqslant b_{n} / 2$. Moreover, since $k_{n}=2 i-1$ for all $n \geqslant 2$, we may also assume that $c_{n}>b_{n} / 2$.

(3.13)Definition. Let $\left[0, \delta\left(\mathscr{P}_{1}, n\right), \Delta\left(\mathscr{P}_{1}, n\right)\right]$ be the remaining interval from the $n$-th step, $n \geqslant 0$. For simplicity, we write $\delta_{n}=\delta\left(\mathscr{P}_{1}, n\right)$ and $\Delta_{n}=\Delta\left(\mathscr{P}_{1}, n\right)$ respectively. At the $(n+1)$-th step, there are $2 i$ tests including the tested point $\delta_{n}$. Denoted them by $0=z_{0}<z_{1}<\cdots<z_{2 i}<z_{2 i+1}=\Delta_{n}$. Then, it follows from the last remark that $\delta_{n}$ must be one of $\left\{z_{i+1}, z_{i+2}, \cdots, z_{2 i}\right\}$. If $\delta_{n}=z_{\ell}$, we say that $\delta_{n}$ is located at the $\ell$-th position.

(3.14)Lemma. Let $\delta_{m-1}$ be located at the $\ell$-th position. Then

$$
\left(\begin{array}{c}
\alpha_{m} \\
\Delta_{m}
\end{array}\right)=\frac{1}{i \chi(\ell-1)-[\ell / 2]}\left(\begin{array}{c}
i \delta_{m-1}-[\ell / 2] \Delta_{m-1} \\
\chi(\ell-1) \Delta_{m-1}-\delta_{m-1}
\end{array}\right)
$$

This occurs only if

$$
\delta_{m-1}>\frac{\ell}{2(i+1)} \Delta_{m-1}, \text { if } \ell=2 j, \quad \delta_{m-1}<\frac{\ell+1}{2(i+1)} \Delta_{m-1}, \text { if } \ell=2 j-1 .
$$

Then, $\delta_{m}=\alpha_{m}$ if and only if

$$
\delta_{m-1}<\frac{\ell}{2 i+1} \Delta_{m-1}, \text { if } \ell=2 j, \quad \delta_{m-1}>\frac{\ell}{2 i+1} \Delta_{m-1}, \text { if } \ell=2 j-1 .
$$

Otherwise,

$$
\delta_{m}=\Delta_{m}-\alpha_{m}=\frac{(\chi(\ell-1)+[\ell / 2]) \Delta_{m-1}-(i+1) \delta_{m-1}}{i \chi(\ell-1)-[\ell / 2]} .
$$

Proof. Since $\delta_{m-1}$ located at the $\ell$-th position, we have

$$
\left(\begin{array}{c}
\delta_{m-1} \\
\Delta_{m-1}
\end{array}\right)=\left(\begin{array}{ll}
\chi(\ell-1) & {[\ell / 2]} \\
1 & i
\end{array}\right)\left(\begin{array}{c}
\alpha_{m} \\
\Delta_{m}
\end{array}\right)
$$

and

$$
\Delta_{m}>\alpha_{m}
$$

Now, (3.15) follows from (3.19). By using (3.15), it is easy to check that (3.16) is equivalent to (3.20). On the other hand, $\delta_{m}=\alpha_{m}$ is equivalent to say that $\alpha_{m}>\Delta_{m} / 2$ by Remark (3.12). Hence, the last two assertions follows by a simple computation.

Let $j_{e}=i / 2+1$ and $j_{0}=(i+1) / 2$ for even and odd $i$ respectively. Define $K(j)=j \Delta_{m-1} /(i+1)$. 
(3.21)Lemma. There are altogether four cases:

i) If $\delta_{m-1} \in(K(j), K(j+1))$ and $\delta_{m-1}$ is located at an even's position $\ell$, then $\ell=2 j$. Where $j$ varies from $j_{e}$ to $i-1$ if $i$ is even; otherwise, from $j_{0}$ to $i-1$.

ii) If $\delta_{m-1} \in(K(j), K(j+1))$ and $\delta_{m-1}$ is located at an odd's position $\ell$, then $\ell=2 j+1$. Where $j$ varies from $j_{e}$ to $i-1$ if $i$ is even; otherwise, from $j_{0}+1$ to $i-1$.

iii) If $\delta_{m-1} \in\left(K(i), \Delta_{m-1}\right)$, then $\delta_{m-1}$ can only be located at the (2i)-th position.

iv) If $\delta_{m-1} \in\left(\Delta_{m-1} / 2, K([(i+3) / 2])\right)$, then $\delta_{m-1}$ can only be located at the $\ell_{0}$-th position with $\ell_{0}=2\left[\frac{i+3}{2}\right]-1=i+1-\chi(i+1)$.

Proof. The partition of sub-intervals $(K(j), K(j+1))$ is suggested by Lemma (3.14), especially (3.16). The range of $j$ 's is due to the fact that the position $\ell$ varies from $i+1$ to $2 i$ and the fact that $\frac{1}{2} \Delta_{m-1}<\delta_{m-1}<\Delta_{m-1}$.

(3.22)Lemma. Given a basic policy $\mathscr{P}_{1}$, assume that at the $m$-th step, we have the $\left[\alpha_{m}, \beta_{m}\right]$-partition.

i) If $\alpha_{1} \in\left(F_{2 n-1} / F_{2 n+1}, G_{2 n-2} / G_{2 n}\right)$, then

$$
\begin{gathered}
\delta\left(\mathscr{P}_{1}, m\right)=(-1)^{m}\left(G_{m-2}-G_{m} \alpha_{1}\right) / i^{m}, \quad 1 \leqslant m \leqslant 2 n-1 \\
\Delta\left(\mathscr{P}_{1}, m\right)=\delta\left(\mathscr{P}_{1}, m-1\right) / i, \quad 2 \leqslant m \leqslant 2 n-1 .
\end{gathered}
$$

Moreover, $\delta\left(\mathscr{P}_{1}, m-1\right)=\alpha_{m}>\beta_{m}$ for all $1 \leqslant m \leqslant 2 n-1$.

ii) If $\alpha_{1} \in\left(G_{2 n-1} / G_{2 n+1}, F_{2 n} / F_{2 n+2}\right)$, then the same assertions in $\left.i\right)$ hold for all $m$ up to $2 n$.

(3.25)Remark. If we set $G_{-2}=1 / i$, then we can keep not only the recurrence $G_{m}=i\left(G_{m-1}+G_{m-2}\right), m \geqslant 0$ but also extend (3.24) to $m=1$, regarding $\delta\left(\mathscr{P}_{1}, 0\right)$ as those given by the right hand side of (3.23). We will use this convention for simplicity. However, we will use this convention only for computing $\delta_{m}$ 's and $\Delta_{m}$ 's with starting value $m=1$. Otherwise, it would contradict to our original convention that $\delta\left(\mathscr{P}_{1}, 0\right)=1 / i$ which is the length of the initial testing interval.

Proof of Lemma (3.22).

a) Let us begin with the first step. We have $\Delta_{1}=\frac{1}{i}\left(\frac{1}{i}-\alpha_{1}\right)>\alpha_{1}$. This gives us one condition $\alpha_{1}<\frac{1}{i(i+1)}=\frac{G_{0}}{G_{2}}$. Clearly, $\delta_{1}=\alpha_{1}$ if and only if $\alpha_{1}>\frac{1}{i(2 i+1)}=\frac{F_{1}}{F_{3}}$. Hence $\alpha_{1} \in\left(\frac{F_{1}}{F_{3}}, \frac{G_{0}}{G_{2}}\right)$.

b) Next, consider the second step. Assume that $\alpha_{1} \in\left(\frac{G_{1}}{G_{3}}, \frac{F_{2}}{F_{4}}\right) \subset\left(\frac{F_{1}}{F_{3}}, \frac{G_{0}}{G_{2}}\right)$. Since in the present situation, $K(i)=\frac{i}{i+1} \Delta_{1}$, by a), it follows that $\delta_{1} \in\left(K(i), \Delta_{1}\right) \Leftrightarrow$ $\alpha_{1} \in\left(\frac{G_{1}}{G_{3}}, \frac{G_{0}}{G_{2}}\right)$. But $\left(\frac{G_{1}}{G_{3}}, \frac{F_{2}}{F_{4}}\right) \subset\left(\frac{G_{1}}{G_{3}}, \frac{G_{0}}{G_{2}}\right)$, hence by Lemma (3.21) we see that $\delta_{1}$ must be located at the (2i)-th position. On the other hand, by (3.17), $\delta_{2}=\alpha_{2}$ is equivalent to $\alpha_{1}<F_{2} / F_{4}$. Thus, we have proved the lemma for $n=1$.

c) Suppose that we now arrive at the $m$-th step. Then $\delta_{m}>K(i)$ means that

$$
\alpha_{m}>i \Delta_{m} /(i+1)=\delta_{m-1} /(i+1) .
$$

Noticing that we have already had $\delta_{m}<\Delta_{m}$ by our assumption, hence by Lemma (3.21), $\delta_{m}$ must be located at the (2i)-th position whenever (3.26) holds. On the other hand, by (3.17), $\delta_{m+1}=\alpha_{m+1}$ if and only if

$$
(2 i+1) \delta_{m}<2 \delta_{m-1}
$$


Furthermore, if these all hold, then $\delta_{m+1}=\alpha_{m+1}=\delta_{m-1} / i-\delta_{m}$. By the hypotheses of induction, we then obtain

$$
\begin{aligned}
\delta_{m+1} & =\frac{(-1)^{m-1}}{i^{m-1}}\left(G_{m-3}-G_{m-1} \alpha_{1}\right)-\frac{(-1)^{m}}{i^{m}}\left(G_{m-2}-G_{m} \alpha_{1}\right) \\
& =\frac{(-1)^{m+1}}{i^{m+1}}\left(G_{m-1}-G_{m+1} \alpha_{1}\right) .
\end{aligned}
$$

This gives (3.23).

We now return to (3.26). By the hypotheses of induction, (3.26) is equivalent to

$$
(i+1)(-1)^{m}\left(G_{m-2}-G_{m} \alpha_{1}\right) / i^{m}>(-1)^{m-1}\left(G_{m-3}-G_{m-1} \alpha_{1}\right) / i^{m-1} .
$$

For odd $m$, this becomes

$$
G_{m-3}+(i+1) G_{m-2} / i<\left(G_{m-1}+(i+1) G_{m} / i\right) \alpha_{1}
$$

By (2.2), this gives us $\alpha_{1}>G_{m} / G_{m+2}$. For even $m,(3.28)$ is equivalent to $\alpha_{1}<$ $G_{m} / G_{m+2}$. On the other hand, for odd and even $m,(3.27)$ gives us

$$
\alpha_{1}<\left(2 G_{m-1}+G_{m-2}\right) /\left(2 G_{m+1}+G_{m}\right)=F_{m+1} / F_{m+3}
$$

and $\alpha_{1}>F_{m+1} / F_{m+3}$ respectively. Combining these facts, we prove the required conclusions.

Observing (2.9), (2.15), (2.20), Lemma (3.11) and Lemma (3.22), it is natural to assume that $\alpha_{1}=\alpha_{1}\left(\mathscr{P}_{1}\right)$ is in one of the following sub-intervals:

$$
\begin{aligned}
& \left(0, \frac{F_{1}}{F_{3}}\right), \quad\left(\frac{F_{2 n-1}}{F_{2 n+1}}, \frac{G_{2 n-1}}{G_{2 n+1}}\right), \quad\left(\frac{G_{2 n-1}}{G_{2 n+1}}, \frac{F_{2 n+1}}{F_{2 n+3}}\right) \\
& \left(\frac{G_{2 n}}{G_{2 n+2}}, \frac{F_{2 n}}{F_{2 n+2}}\right), \quad\left(\frac{F_{2 n}}{F_{2 n+2}}, \frac{G_{2 n-2}}{G_{2 n}}\right), \quad\left(\frac{G_{0}}{G_{2}}, \frac{1}{i}\right), \quad n \geqslant 1 .
\end{aligned}
$$

The first three are contained in $\left[0, \omega^{2}\right]$ but the second three in $\left[\omega^{2}, 1 / i\right]$. However, as we have seen from the proof a) of Lemma (3.22), $\alpha_{1}<G_{0} / G_{2}$. Thus, the last one in (3.29) can be ignored. Now, we want to prove that the second one for $n \geqslant 2$ and the third one for all $n$ can also be ignored.

(3.30)Lemma. Let $m \geqslant 1$ and $\delta_{m}=(-1)^{m}\left(G_{m-2}-G_{m} \alpha_{1}\right) / i^{m}$. Then $F_{m+2} \delta_{m}<$ $\delta$ if and only if

$$
(-1)^{m-1} \alpha_{1}<\left\{i^{m} \delta / F_{m+2}+(-1)^{m-1} G_{m-2}\right\} / G_{m}=:(-1)^{m-1} A_{m} .
$$

In particular, $A_{1}=\delta / F_{3}=F_{4} \omega^{3} / F_{3}$ and $A_{2}=\omega^{2}$.

Proof. The assertions follow by some simple computations. For instance, let $m=2$, then

$$
\alpha_{1}>\frac{1}{G_{2}}\left(G_{0}-\frac{i^{2} \delta}{F_{4}}\right)=\frac{1}{i(i+1)}\left\{1-i^{2} \omega^{3}\right\}=\frac{1}{i(i+1)}\{1-i \omega(1-i \omega)\}=\omega^{2} .
$$

This shows that $A_{2}=\omega^{2}$. 
By Lemma $(3.22)$, if $\alpha_{1} \in\left(\frac{F_{2 n-1}}{F_{2 n+1}}, \frac{G_{2 n-1}}{G_{2 n+1}}\right)$ with $n \geqslant 2$ or $\alpha_{1} \in\left(\frac{G_{2 n-1}}{G_{2 n+1}}, \frac{F_{2 n+1}}{F_{2 n+3}}\right)$ with $n \geqslant 1$, then the assumption of Lemma (3.30) holds for $m=1$ and 2 . Hence $F_{4} \delta_{2} \geqslant \delta$ and so (3.9) holds. Thus, the proof of our main theorem is done for these cases. Therefore, we need only to consider the cases that $\alpha_{1}$ is in one of the sub-intervals:

$$
\left(0, \frac{F_{1}}{F_{3}}\right), \quad\left(\frac{F_{1}}{F_{3}}, \frac{G_{1}}{G_{3}}\right), \quad\left(\frac{G_{2 n}}{G_{2 n+2}}, \frac{F_{2 n}}{F_{2 n+2}}\right), \quad\left(\frac{F_{2 n}}{F_{2 n+2}}, \frac{G_{2 n-2}}{G_{2 n}}\right) .
$$

Suppose that $\alpha_{1}$ is in the second interval. Then, by Lemma (3.22), the explicit expression for $\delta\left(\mathscr{P}_{1}, m\right)$ works only for $m=1$. If we could find an $n_{0}$ so that

$$
F_{n_{0}+2} \delta\left(\mathscr{P}_{1}, n_{0}\right) \geqslant \delta
$$

Then, we were done. The problem is that such an $n_{0}$ for which (3.33) holds can be very large. And there is no simple way to find out $n_{0}$ since there is no simple expression for $\delta\left(\mathscr{P}_{1}, m\right)$ when $m>1$. Because of this, we employ the limiting behavior of $F_{n+2} \delta\left(\mathscr{P}_{1}, n\right)$ as $n \rightarrow \infty$. And this is just what we are going to study in the next section.

\section{Optimal Policy at Infinity.}

In this section, we study the optimal policy at infinity. The results obtained here are not only for the later use but also have their own interesting. For our reader's convenience, we first copy some lemmas from [5] which are available for any sequence $k_{1} \geqslant 2, k_{2}, \cdots$ of positive integers. Let $\left(\begin{array}{l}x_{n} \\ y_{n}\end{array}\right)$ be the solution to the equations

$$
\left(\begin{array}{l}
x_{n} \\
y_{n}
\end{array}\right)=c\left(k_{n+1}\right)\left(\begin{array}{l}
x_{n+1} \\
y_{n+1}
\end{array}\right), n \geqslant 0, \quad x_{0}=b-a>0
$$

and $\left(\begin{array}{l}u_{n} \\ v_{n}\end{array}\right)$ satisfies

$$
\left(\begin{array}{l}
u_{n} \\
v_{n}
\end{array}\right) \leqslant c\left(k_{n+1}\right)\left(\begin{array}{l}
u_{n+1} \\
v_{n+1}
\end{array}\right), n \geqslant 0, \quad u_{0}=b-a
$$

Put

$$
\mu(m, n)=\frac{v_{n}}{y_{n}} / \frac{u_{m}}{x_{m}}, \quad \lambda(m, n)=\frac{v_{n}}{y_{n}} / \frac{v_{m}}{y_{m}}, \quad \rho(m, n)=\frac{u_{n}}{x_{n}} / \frac{u_{m}}{x_{m}} .
$$

Obviously, we have

$$
\begin{array}{lll}
\text { (4.4)Lemma }^{[5]} \cdot & \lambda(m, l) \lambda(l, n)=\lambda(m, n), \quad \rho(m, l) \rho(l, n)=\rho(m, n), \\
& \mu(m, l) \lambda(l, n)=\mu(m, n), \quad \rho(m, l) \mu(l, n)=\mu(m, n), m, n, l \geqslant
\end{array}
$$

0 .

(4.5)Lemma ${ }^{[5]}$. If $k_{n+1}=2 i$, then $\lambda(n, n+1) \geqslant 1$ and $\mu(n, n+1) \geqslant i /(i+1)$. If $k_{n+1}=2 i-1$, then $\lambda(n, n+1) \geqslant i /(i+1)$ and $\mu(n, n+1) \geqslant 1$. 
(4.6)Lemma ${ }^{[5]}$. If $\lambda(n, n+1)<1$, then $\mu(n+1, n)^{-1} \geqslant \lambda(n, n+1)^{-1}$.

(4.7)Lemma ${ }^{[5]}$. If $\mu(n, n+1)<1$, then $\rho(n, n+1) \geqslant \mu(n, n+1)^{-1}$.

Now, we return to our main setup: $k_{1} \geqslant 2, k_{n}=2 i-1$ for all $n \geqslant 2$. In this case, by Lemma (4.5), we have

$$
\lambda(n, n+1) \geqslant i /(i+1) \text { and } \mu(n, n+1) \geqslant 1 \text { for all } n \geqslant 2 .
$$

(4.9)Lemma. For each $n \geqslant 0$, if $\lambda(n, n+1)<1$, then $\lambda(n, n+2) \geqslant \lambda(n, n+1)^{-1}>$ 1.

Proof. By Lemma (4.4), it follows that $\mu(n+1, n) \lambda(n, n+2)=\mu(n+1, n+2)$. Hence, by Lemma (4.6), we have $\lambda(n, n+2)=\mu(n+1, n)^{-1} \mu(n+1, n+2) \geqslant$ $\mu(n+1, n)^{-1} \geqslant \lambda(n, n+1)^{-1}$.

(4.10)Lemma ${ }^{[5]}$. Let $\sigma=(b-a) /\left\{\chi\left(k_{1}\right) \omega+\left[\frac{k_{1}+1}{2}\right] / i\right\}$. Then $x_{n}=\sigma \omega^{n}, y_{n}=$ $\sigma \omega^{n-1} / i, n \geqslant 1$ is the unique solution to the equations

$$
\left(\begin{array}{l}
x_{n} \\
y_{n}
\end{array}\right)=c\left(k_{n+1}\right)\left(\begin{array}{l}
x_{n+1} \\
y_{n+1}
\end{array}\right), n \geqslant 0, \quad x_{0}=b-a .
$$

From now on, unless otherwise stated, let $\mathscr{P}_{2}$ to denote an arbitrary policy with initial testing interval $[a, b]$ and with successive testing numbers $k_{1} \geqslant 2, k_{n}=2 i-1$ for all $n \geqslant 2$. Let $\mathscr{H}_{2}$ denote the basic policy: at the $n$-th step, the $\left[\alpha_{n}, \beta_{n}\right]$ partition is determined by $\alpha_{n}=x_{n}, \beta_{n}=y_{n}-x_{n}, n \geqslant 1$, where $\left(x_{n}, y_{n}\right)$ is given by Lemma (4.10). For simplicity, we put $u_{n}=\delta\left(\mathscr{P}_{2}, n\right), v_{n}=\Delta\left(\mathscr{P}_{2}, n\right), n \geqslant 0$ where $u_{0}=\delta\left(\mathscr{P}_{2}, 0\right)=b-a$. It is known that $\left(\begin{array}{l}u_{n} \\ v_{n}\end{array}\right)$ satisfies $(4.2)^{[5]}$.

Actually, the policies $\mathscr{P}_{2}$ and $\mathscr{H}_{2}$ are the generalization of $\mathscr{P}_{1}$ and $\mathscr{H}_{1}$ respectively. If we take $[a, b]=[0,1 / i]$ and $k_{1}=2 i$, then $\mathscr{P}_{2}$ and $\mathscr{H}_{2}$ coincide with $\mathscr{P}_{1}$ and $\mathscr{H}_{1}$ respectively. But we prefer to distinguish them.

Next, we introduce a sequence $\left\{\varphi_{j}\right\}$ by the following procedure.

(I) Let $k_{1}$ be an odd integer. In this case, we always have $\mu(0,1) \geqslant 1$. If $\mu(0,1)>1$, we simply take $\varphi_{1}=\mu(0,1)$. Otherwise, we look at the sequence $\{\lambda(n, n+1): n \geqslant 1\}$. If $\lambda(n, n+1) \geqslant 1$ for all $n \geqslant 1$, we cancel those $\lambda(n, n+1)$ which equals one and denote by $\varphi_{1}, \varphi_{2}, \cdots$ the remaining $\lambda(n, n+1)$ 's successively. Then the construction is done. Conversely, if we find some $\lambda(n, n+1)<1$. Then, by Lemma (4.9), we have $\lambda(n, n+2) \geqslant \lambda(n, n+1)^{-1}>1$. In this case, we will forget $\lambda(n, n+1)$ and $\lambda(n+1, n+2)$ and take $\lambda(n, n+2)$ as one of the $\varphi$ 's. Then go ahead to look at $\{\lambda(m, m+1): m \geqslant n+2\}$ and repeat the same procedure. Of course, the index set $J$ of $\left\{\varphi_{j}\right\}$ may be empty, which is equivalently to say that $\mu(0,1)=1$ and $\lambda(n, n+1)=1$ for all $n \geqslant 1$.

(II) Let $k_{1}$ be an even integer. If $\mu(0,1) \geqslant 1$, then we can adopt the same construction for $\varphi$ as given in (I). But in this case, it can be happen that $\mu(0,1)<1$ for which we have to modify the above construction. By Lemma (4.7), we then have $\rho(0,1) \geqslant \mu(0,1)^{-1}>1$. And so we set $\varphi_{1}=\rho(0,1)>1$. Now, we have $\mu(1,2) \geqslant 1$. This enables us to return to the previous construction by regarding $\mu(1,2)$ and $\{\lambda(n, n+1): n \geqslant 2\}$ as $\mu(0,1)$ and $\{\lambda(n, n+1): n \geqslant 1\}$ respectively. Again, the index set $J$ of $\left\{\varphi_{j}\right\}$ is empty if and only if $\mu(0,1)=\lambda(n, n+1)=1$ for all $n \geqslant 1$. 
To get a precise impression of the above construction, consider a special case: $\mu(0,1)<1, \mu(1,2)>1, \lambda(2,3)=1, \lambda(3,4)<1$ and $\lambda(n, n+1)=1$ for all $n \geqslant 5$. Then we have $\varphi_{1}=\rho(0,1), \varphi_{2}=\mu(1,2), \varphi_{3}=\lambda(3,5)$ and $J=\{1,2,3\}$. On the other hand, by Lemma (4.4), we see that

$$
\begin{gathered}
\frac{\Delta\left(\mathscr{P}_{2}, n\right)}{\Delta\left(\mathscr{H}_{2}, n\right)}=\frac{v_{n}}{y_{n}}=\frac{v_{n}}{y_{n}} / \frac{u_{0}}{x_{0}}=\mu(0, n)=\rho(0,1) \mu(1, n)=\rho(0,1) \mu(1,2) \lambda(2, n) \\
=[\rho(0,1)][\mu(1,2)] \lambda(2,3)[\lambda(3,4) \lambda(4,5)] \lambda(5,6) \cdots \lambda(n-1, n) \geqslant \prod_{j=1}^{3} \varphi_{j}, \\
n \geqslant 5 .
\end{gathered}
$$

This example not only shows the reason why we introduced such a construction for $\varphi^{\prime}$ s but also indicates the proof of the following result.

(4.11)Proposition ${ }^{[4,5]}$. For any $\mathscr{P}_{2}$, we have $\lim _{n \rightarrow \infty} \frac{\Delta\left(\mathscr{P}_{2}, n\right)}{\Delta\left(\mathscr{H}_{2}, n\right)} \geqslant \prod_{j} \varphi_{j} \geqslant 1$. Moreover, the equality holds if and only if $\mu(0,1)=\lambda(n, n+1)=1$ for all $n \geqslant 1$.

We are now ready to prove the main result of this section.

(4.12)Theorem. $\mathscr{H}_{2}$ is the optimal policy at infinity. That is, for any policy $\mathscr{P}_{2}$, we have

$$
\lim _{n \rightarrow \infty} \frac{\delta\left(\mathscr{P}_{2}, n\right)}{\delta\left(\mathscr{H}_{2}, n\right)}=\lim _{n \rightarrow \infty} \frac{\Delta\left(\mathscr{P}_{2}, n\right)}{\Delta\left(\mathscr{H}_{2}, n\right)}=\prod_{j} \varphi_{j} \geqslant 1
$$

Proof. Write $\delta\left(\mathscr{P}_{2}, n\right) / \delta\left(\mathscr{H}_{2}, n\right)$ as

$$
\frac{\delta\left(\mathscr{P}_{2}, n\right)}{\delta\left(\mathscr{H}_{2}, n\right)}=\frac{\Delta\left(\mathscr{P}_{2}, n\right)}{\Delta\left(\mathscr{H}_{2}, n\right)} \cdot \frac{\delta\left(\mathscr{P}_{2}, n\right)}{\Delta\left(\mathscr{P}_{2}, n\right) i \omega}=D_{n} \frac{\xi_{n}}{i \omega},
$$

where

$$
D_{n}=\frac{\Delta\left(\mathscr{P}_{2}, n\right)}{\Delta\left(\mathscr{H}_{2}, n\right)}, \quad \xi_{n}=\frac{\delta\left(\mathscr{P}_{2}, n\right)}{\Delta\left(\mathscr{P}_{2}, n\right)} .
$$

Here, we have used the fact that $\delta\left(\mathscr{H}_{2}, n\right)=\sigma \omega^{n}=\left(\sigma \omega^{n-1} / i\right) \cdot i \omega=\Delta\left(\mathscr{H}_{2}, n\right) i \omega$ as given in Lemma (4.10). By Proposition (4.11), the $\operatorname{limit}_{\lim } \lim _{n} D_{n}$ exists. If $\lim _{n} D_{n}=\infty$, then it follows from $\xi_{n} \geqslant 1 / 2$ that

$$
\lim _{n \rightarrow \infty} \frac{\delta\left(\mathscr{P}_{2}, n\right)}{\delta\left(\mathscr{H}_{2}, n\right)}=\lim _{n \rightarrow \infty} \frac{\Delta\left(\mathscr{P}_{2}, n\right)}{\Delta\left(\mathscr{H}_{2}, n\right)} .
$$

Hence, we may and will assume that $\lim _{n \rightarrow \infty} D_{n}<\infty$. But then, from (4.11) and the construction of $\varphi_{j}$ 's, we must have

$$
\frac{\Delta\left(\mathscr{P}_{2}, n+1\right)}{\Delta\left(\mathscr{P}_{2}, n\right) \omega}=\lambda(n, n+1) \longrightarrow 1 \quad \text { as } n \rightarrow \infty .
$$

On the other hand, as we have mentioned above, for any policy $\mathscr{P}_{2}$, we have

$$
\left(\begin{array}{c}
\delta\left(\mathscr{P}_{2}, n\right) \\
\Delta\left(\mathscr{P}_{2}, n\right)
\end{array}\right) \leqslant\left(\begin{array}{cc}
0 & i \\
1 & i
\end{array}\right)\left(\begin{array}{c}
\delta\left(\mathscr{P}_{2}, n+1\right) \\
\Delta\left(\mathscr{P}_{2}, n+1\right)
\end{array}\right) .
$$


In particular,

$$
\left(i+\xi_{n}\right) \lambda(n-1, n) \omega=\left(i+\frac{\delta\left(\mathscr{P}_{2}, n\right)}{\Delta\left(\mathscr{P}_{2}, n\right)}\right) \frac{\Delta\left(\mathscr{P}_{2}, n\right)}{\Delta\left(\mathscr{P}_{2}, n-1\right)}=\frac{\delta\left(\mathscr{P}_{2}, n\right)+i \Delta\left(\mathscr{P}_{2}, n\right)}{\Delta\left(\mathscr{P}_{2}, n-1\right)} \geqslant 1
$$

Hence

$$
\frac{\xi_{n}}{i \omega} \geqslant \frac{1-i \lambda(n-1, n) \omega}{i \lambda(n-1, n) \omega^{2}}
$$

From this and (4.15), we get

$$
\underline{\lim }_{n \rightarrow \infty} \xi_{n} /(i \omega) \geqslant(1-i \omega) /\left(i \omega^{2}\right)=1
$$

On the other hand, by (4.16) again, we have

$$
\frac{\delta\left(\mathscr{P}_{2}, n\right)}{\delta\left(\mathscr{H}_{2}, n\right)} \leqslant \frac{i \Delta\left(\mathscr{P}_{2}, n+1\right)}{\delta\left(\mathscr{H}_{2}, n\right)}=\frac{\Delta\left(\mathscr{P}_{2}, n+1\right)}{\Delta\left(\mathscr{H}_{2}, n+1\right)}
$$

Combining this with (4.13) and (4.17), we finally arrive at

$$
\begin{aligned}
\lim _{n} D_{n} & \leqslant\left(\lim _{n} D_{n}\right) \underline{\lim }_{n} \frac{\xi_{n}}{i \omega} \\
& \leqslant \underline{\lim }_{n} \frac{\delta\left(\mathscr{P}_{2}, n\right)}{\delta\left(\mathscr{H}_{2}, n\right)} \\
& \leqslant \varlimsup_{n} \frac{\delta\left(\mathscr{P}_{2}, n\right)}{\delta\left(\mathscr{H}_{2}, n\right)} \\
& \leqslant \lim _{n} \frac{\Delta\left(\mathscr{P}_{2}, n+1\right)}{\Delta\left(\mathscr{H}_{2}, n+1\right)} \\
& =\lim _{n} D_{n} .
\end{aligned}
$$

Therefore, we claim that $\lim _{n} \xi_{n} /(i \omega)=1$ and hence (4.14) holds.

To conclude this section, we show that the optimal policy at infinity is essentially unique.

(4.18)Corollary. If $\mathscr{P}_{2}$ is not equivalent to $\mathscr{H}_{2}$, then we have $\lim _{n \rightarrow \infty} \frac{\delta\left(\mathscr{P}_{2}, n\right)}{\delta\left(\mathscr{H}_{2}, n\right)}>1$.

Proof. The conclusion follows from Proposition (4.11) and Theorem (4.12) immediately.

\section{Proof of Theorem (1.15).}

We begin this section by introducing a comparison lemma. Suppose that we are now at the $N$-th step. Then the policy $\mathscr{P}_{1}$ corresponds in a natural way a basic policy $\mathscr{P}_{2}$ having successive testing numbers $k_{1}=2 i, k_{n}=2 i-1$ for all $n \geqslant 2$ and initial testing interval $\left[0, \Delta_{N-1}\right]$. Moreover, $\delta\left(\mathscr{P}_{1}, N+m-1\right)=\delta\left(\mathscr{P}_{2}, m\right), m \geqslant 1$. Recalling that for the policy $\mathscr{H}_{2}$ defined by Lemma $(4.10)$, we have $\delta\left(\mathscr{H}_{2}, m\right)=$ $\sigma \omega^{m}$, where

$$
\sigma=\Delta_{N-1} .
$$


As an application of Theorem (4.12), we obtain

$$
\begin{aligned}
\sup _{m \geqslant 1} F_{m+2} \delta\left(\mathscr{P}_{1}, m\right) & \geqslant \lim _{m \rightarrow \infty} F_{m+2} \delta\left(\mathscr{P}_{1}, m\right)=\lim _{m \rightarrow \infty} F_{N+m+1} \delta\left(\mathscr{P}_{1}, N+m-1\right) \\
& =\lim _{m \rightarrow \infty}\left(\frac{F_{N+m+1}}{F_{m+1}} F_{m+1} \delta\left(\mathscr{P}_{2}, m\right)\right)=\frac{1}{\omega^{N}} \lim _{m \rightarrow \infty} F_{m+1} \delta\left(\mathscr{P}_{2}, m\right) \\
& \geqslant \omega^{-N} \lim _{m \rightarrow \infty} F_{m+1} \delta\left(\mathscr{H}_{2}, m\right)=\sigma \omega^{-N} \lim _{m \rightarrow \infty} F_{m+1} \omega^{m} .
\end{aligned}
$$

Thus, we have proved the following result:

(5.3)Lemma. Let $\gamma=F_{4} \omega^{3} / \lim _{n \rightarrow \infty} F_{n+1} \omega^{n}$. Then, we have $\delta\left(\mathscr{P}_{1}\right) \geqslant \delta$ provided

$$
\sigma \omega^{-N} \geqslant \gamma
$$

Based on this lemma, we can now make a complement to Lemma (3.30).

(5.5)Lemma. Let $m \geqslant 1$ and $\Delta_{m}=(-1)^{m-1}\left(G_{m-3}-G_{m-1} \alpha_{1}\right) / i^{m}$. Then $\delta\left(\mathscr{P}_{1}\right)$ $<\delta$ only if

$$
(-1)^{m} \alpha_{1}<\left\{i^{m} \omega^{m+1} \gamma+(-1)^{m} G_{m-3}\right\} / G_{m-1}=: B_{m}
$$

Proof. Applying Lemma (5.3) to the case that $N=m+1$, we obtain

$$
\sigma \omega^{-N}=\Delta_{m} / \omega^{m+1}=(-1)^{m-1}\left(G_{m-3}-G_{m-1} \alpha_{1}\right) /\left(i^{m} \omega^{m+1}\right) .
$$

Thus, $\sigma \omega^{-N} \geqslant \gamma$ is equivalent to $(-1)^{m} \alpha_{1} \leqslant\left\{i^{m} \omega^{m+1} \gamma+(-1)^{m} G_{m-3}\right\} / G_{m-1}$. This proves our assertion.

Consider the special case that $m=1$. That is

$$
\Delta_{1}=\left(1 / i-\alpha_{1}\right) / i
$$

Then, the condition (5.6) becomes $\alpha_{1} \geqslant \frac{1}{i}-i \gamma \omega^{2}>\frac{1}{i}-\gamma \omega$. But we have

$$
1 / i-\gamma \omega>G_{1} / G_{3}
$$

The proofs of this and some subsequent elementary inequalities are delayed to the end of this section for keeping the main line of the proof of Theorem (1.15).

Because (5.7) holds for any choice of $\alpha_{1}$, the above facts enable us to remove the first two sub-intervals in (3.32). Thus, for the rest of the proof, we need only to consider the intervals:

$$
\left(\frac{G_{2 n}}{G_{2 n+2}}, \frac{F_{2 n}}{F_{2 n+2}}\right), \quad\left(\frac{F_{2 n}}{F_{2 n+2}}, \frac{G_{2 n-2}}{G_{2 n}}\right) .
$$

Now, we are at the position to complete the proof of Theorem (1.15). Note that $\mu(0,1)=\Delta\left(\mathscr{P}_{1}, 1\right) / \Delta\left(\mathscr{H}_{1}, 1\right)=i \Delta_{1} / \omega$. Thus, $\alpha_{1}>\omega^{2} \Leftrightarrow \mu(0,1)<1$. If $\mu(0,1)^{-1} \geqslant \gamma$, then $\varphi_{1} \geqslant \gamma$ and there is nothing to do. We assume that $\mu(0,1)^{-1}<$ $\gamma$. Equivalently, $\alpha_{1}<\frac{1}{i}-\frac{\omega}{\gamma}$. But as we will prove later (Lemma (5.20)) that

$$
1 / i-\omega / \gamma<F_{2} / F_{4} .
$$


This means that we do not need to consider the sub-interval $\left(F_{2} / F_{4}, G_{0} / G_{2}\right)$. Furthermore, by Lemma (3.22), for

$$
\alpha_{1} \in\left(\frac{G_{2 n}}{G_{2 n+2}}, \frac{F_{2 n}}{F_{2 n+2}}\right), \quad n \geqslant 1
$$

or

$$
\alpha_{1} \in\left(\frac{F_{2 n}}{F_{2 n+2}}, \frac{G_{2 n-2}}{G_{2 n}}\right), \quad n \geqslant 2
$$

the formulas of $\delta_{m}$ and $\Delta_{m}$ given by (3.23) and (3.24) are available at least for $m \leqslant 2$. In particular, $\mu(0,1)=\Delta\left(\mathscr{P}_{1}, 2\right) / \Delta\left(\mathscr{H}_{1}, 2\right)=\alpha_{1} / \omega^{2}$. Hence, the proof is deduced to consider the case that $\omega^{2}<\alpha_{1}<\gamma \omega^{2}$. Given such an $\alpha_{1}$, there exists uniquely an $n_{0}$ so that one of (5.11) and (5.12) holds. We now discuss these two cases separately.

(I) Let (5.12) hold for some $n_{0} \geqslant 2$. Then by Lemma (3.22) and Lemma (3.30), we have $F_{2 n_{0}+1} \delta_{2 n_{0}-1} \geqslant \delta$ unless $\alpha_{1}<A_{2 n_{0}-1}$. We now prove that this is impossible. This follows once we prove that $A_{2 n_{0}-1}<F_{2 n_{0}} / F_{2 n_{0}+2}$ which contradicts to our assumption. To do so, noticing that the last inequality is equivalent to $\delta<F_{2} F_{2 n_{0}+1} / F_{2 n_{0}+2}$, by (2.8), we need only to show that

$$
\delta<F_{2} F_{5} / F_{6}
$$

We will check this in Lemma (5.15).

(II) Let (5.11) hold for some $n_{0} \geqslant 1$. Then, we have $F_{2 n_{0}+2} \delta_{2 n_{0}} \geqslant \delta$ unless $\alpha_{1}<B_{2 n_{0}}$. But we can prove that $B_{2 n_{0}}<G_{2 n_{0}} / G_{2 n_{0}+2}$. This again gives us a contradiction. Actually, the above inequality is equivalent to $\omega^{2 n_{0}+1} G_{2 n_{0}+2}<$ $(i+1) / \gamma$. Hence, it suffices to show that

$$
\omega^{3} G_{4}<(i+1) / \gamma
$$

This will be done by Lemma (5.19). Finally, we conclude our main proof by the following four lemmas.

(5.15)Lemma. $\delta<F_{2} F_{5} / F_{6}$.

Proof. By $(3.4), \delta=i(2 i+3)((i+1) \omega-1)$. We need only to show that $(i+1) \omega<$ $1+\frac{2 F_{5}}{(2 i+3) F_{6}}$. But the right hand side equals to

$$
\begin{aligned}
1+\frac{2}{2 i+3} \cdot \frac{2 i^{2}+5 i+1}{i\left(2 i^{2}+7 i+4\right)} & =1+\frac{2}{i(2 i+3)}\left(1-\frac{2 i+3}{2 i^{2}+7 i+4}\right) \\
& =1+\frac{2}{i(2 i+3)}-\frac{2}{i\left(2 i^{2}+7 i+4\right)} .
\end{aligned}
$$

On the other hand,

$$
(i+1) \omega<\frac{(i+1) F_{6}}{F_{7}}=\frac{(i+1)\left(2 i^{2}+7 i+4\right)}{2 i^{3}+9 i^{2}+9 i+1}=1+\frac{2 i+3}{2 i^{3}+9 i^{2}+9 i+1}<1+\frac{1}{i(i+3)} .
$$

Thus, it suffices to show that $\frac{2}{2 i^{2}+7 i+4}<\frac{2}{2 i+3}-\frac{1}{i+3}=\frac{3}{2 i^{2}+9 i+9}$. This certainly holds for all $i \geqslant 2$. 
(5.17)Lemma. $1 / i-\gamma \omega>G_{1} / G_{3}$.

Proof. Observe that the assertion is equivalent to

$$
\gamma<(i+1)(1+\omega) /(i+2) \text {. }
$$

By (3.2) and (3.3), this becomes $\frac{i(i+4)(2 i+3)((i+1) \omega-1)}{2(i+1)+3 i \omega}<\frac{i+1}{i+2}(1+\omega)$. That is

$$
\begin{aligned}
i(i+2)(i+4)(2 i+3)((i+1) \omega-1) & <(i+1)(1+\omega)(2(i+1)+3 i \omega) \\
& =(i+1)(2 i+5+2(i+1) \omega) .
\end{aligned}
$$

Or

$\omega<\frac{(i+1)(2 i+5)+i(i+2)(i+4)(2 i+3)}{(i+1)(i(i+2)(i+4)(2 i+3)-2(i+1))}=\frac{2 i^{4}+15 i^{3}+36 i^{2}+31 i+5}{2 i^{5}+17 i^{4}+49 i^{3}+56 i^{2}+20 i-2}$.

Note that the right hand side is greater than $1 /(i+1-1 / i)$ and $\omega=(\sqrt{1+4 / i}-1) / 2$. Now, it should be easy to obtain the required assertion.

(5.19)Lemma. $\omega^{3} G_{4}<(i+1) / \gamma$.

Proof. It follows from (5.16) that

$$
G_{4} \omega^{3}=i^{2}\left(i^{2}+3 i+1\right) \omega^{3}=i\left(i^{2}+3 i+1\right)((i+1) \omega-1)<\left(i^{2}+3 i+1\right) /(i+3) .
$$

Thus, it suffices to show that $\gamma<\frac{(i+1)(i+3)}{i^{2}+3 i+1}=1+\frac{i+2}{i^{2}+3 i+1}$. But this follows from (5.18) and (5.16):

$$
\gamma<(1-1 /(i+2))(1+\omega)=1+((i+1) \omega-1) /(i+2)<1+1 /(i+1) .
$$

(5.20)Lemma. $1 / i-\omega / \gamma<F_{2} / F_{4}$.

Proof. The assertion is the same as follows: $\gamma<i(2 i+3) \omega /(2 i+1)$. By (5.18), it is enough to show that $\frac{i+1}{i+2}(1+\omega)<\frac{i(2 i+3)}{2 i+1} \omega$. Equivalently, $\omega>\frac{2 i^{2}+3 i+1}{2 i^{3}+5 i^{2}+3 i-1}$. Note that the right hand side is less than $1 /(i+1-1 /(2 i-1))$, it is now easy to complete the proof.

\section{REFERENCES}

[1]. M.F.Chen, Optimal method for block-search without knowing the number of experiments (In Chinese), Guiyang Teacher's College, J. (1977), no. 3, 117-134.

[2]. M.F.Chen, On "optimization with comparison tests" (In Chinese), Beijing Normal Univ. J. (1979), no. 3, 66-73.

[3]. J.Kiefer, Sequential minimax search for a maximum, Proc. Amer. Math. Soc. (1953), no. 4, 502-506.

[4]. J.W.Hong, On the optimality of the golden section search (In Chinese), Practice and Study of Mathematics (1973), no. 2, 34-41.

[5]. J.W.Hong, On one-dimensional optimal policy without restrict the number of experiments (In Chinese), Sci. Sin. (1974), no. 2.

[6]. L.K.Hua, Introduction to Optimization in Popular Language (In Chinese), Science Press, Beijing, 1971.

[7]. L.K.Hua, Optimization (In Chinese), Science Press, Beijing, 1981.

[8]. D.J.Wilde and C.S.Beightler, Foundations of Optimization, Prentice-Hall, Inc., 1967.

Department of Mathematics, Beijing Normal University, Beijing 100875.

Department of Mathematics, Fujian Normal University, Fujian Province 350007. 